\title{
Remotely Powered Implantable Heart Monitoring System for Freely Moving Animals
}

\author{
Enver G. Kilinc ${ }^{\star}$, Alejandro C. Moya ${ }^{\star}$, Harald van Lintel*, \\ Philippe Renaud*, Franco Maloberti`, Qing Wang ${ }^{\bullet}$ and Catherine Dehollain* \\ ${ }^{\star}$ RF-IC Group, EPFL, Lausanne, Switzerland, * Microsystems Laboratory LMIS4, EPFL, Lausanne, Switzerland \\ $\diamond$ Integrated Microsystem Laboratory, Universitá degli Studi di Pavia, Pavia, Italy \\ - Division of Nephrology and Hypertension, Department of Medicine, CHUV, Lausanne, Switzerland \\ ${ }^{\circ}$ Huazhong University of Science and Technology, Wuhan, China \\ Email: enver.kilinc@epfl.ch, qing.wang@chuv.ch, catherine.dehollain@epfl.ch
}

\begin{abstract}
This paper presents a remotely powered implantable heart monitoring system for freely moving animals. The system measures the blood pressure in the left ventricle of the heart and transmits the data to a database unit. The implanted unit is remotely powered over $25 \mathrm{~mm}$ at $8 \mathrm{MHz}$ and has autonomous power control system for changing received power levels due to the moving animal. The blood pressure is measured by a piezoresistive sensor die. The data is transmitted by a OOK modulated transmitter at $868 \mathrm{MHz}$. The system is realized by using discrete components which are available on the market. The overall size of the implanted unit is $26 \times 13 \times 5.5 \mathrm{~mm}$ and the overall power consumption is around $7 \mathrm{~mW}$. Experimental results show the effectiveness of the implantable monitoring system.
\end{abstract}

\section{INTRODUCTION}

Today, approximately $25 \%$ of the adult population worldwide are hypertensive [1]. Hypertension is the most important risk factor leading to cardiovascular diseases (CVD) and causing $60 \%$ of stroke and $50 \%$ of myocardial infarction (or coronary heart disease). CVD is responsible for approximately one-third of deaths worldwide and will rise from 17.1 million in 2004 to 23.4 million in 2030 [2]. Monitoring blood pressure and heart function are useful parameters for predicting cardiovascular and cerebrovascular events and for treatment on patients with hypertension and cardiovascular diseases. Today, small rodent animals (mice and rats) are widely used for studying pathogenesis of human hypertension, cardiovascular diseases, diabetes etc. in order to develop new diagnosis and therapeutic strategies.

The implantable wireless sensor systems for the small animals become more widespread [3], [4]. However, design of the implanted device has difficult challenges due to the small size of the animal. The implanted device needs to be small and light-weighted for implantation in the animal. In general, animals are anesthetized during the measurement. Anesthesia has negative impact on blood pressure and heart function [5]. Accordingly, conscious measurements without anesthesia, particularly measurements in a freely moving animal can bring reliable results.

This paper presents an implantable heart monitoring system for a freely moving animal. The implantable system follows the blood pressure in the left ventricle of the heart which gives important information of heart function. The goal is to

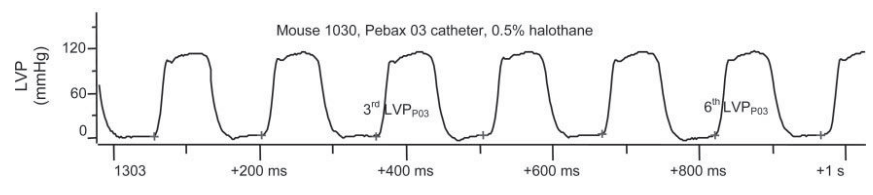

Fig. 1. Example of tracing of LVP from small animal [5]

achieve the implantable heart function monitory system that can be applied to animals with hypertension and heart failure. The system monitors the left ventricular systolic pressure $(L V-S P)$, the maximum rates of $\mathrm{LV}$ pressure rise and fall $\left(L V d P / d t_{\max }\right.$ and $L V d P / d t_{\min }$, determine heart systolic and diastolic functions), and the left ventricular end diastolic pressure ( $L V-E D P$, determines heart diastolic function) in order to determine heart function state and treatment [5], [6].

\section{Overview of Heart Monitoring System}

Many treatments are developed by using small rodent animals. Also, some treatments can be achieved for the hypertension and heart failure. In order to prevent heart failure and have better treatment for hypertension, a long-term cardiovascular hemodynamic monitoring is necessary. Fig. 1 illustrates an example of tracing of Left Ventricular Pressure (LVP) from a small rodent animal. The tracing shows LVP signal in which $L V d P / d t_{\max }$ and $L V d P / d t_{\min }, L V-E D P$, etc. are important parameters for determination of heart function. However, the LVP signal should be monitored for long-term. Accordingly, the long-term monitoring on freely moving small animal introduces also difficulties to be solved.

Firstly, the LVP signal of a small animal needs higher data rate since the small animal has higher heart rate than the humans. In addition, the power should be transmitted to the implanted system inside a freely moving animal for long-term monitoring. Fig. 2 demonstrates a scenario for remotely powered implantable heart monitoring system for a freely moving animal. The implanted monitoring system has a pressure sensor to measure the blood pressure in the left ventricle of the heart. The measured data is transmitted to a receiver and transferred to the database unit. The implanted system is remotely powered by using magnetic coupling which 


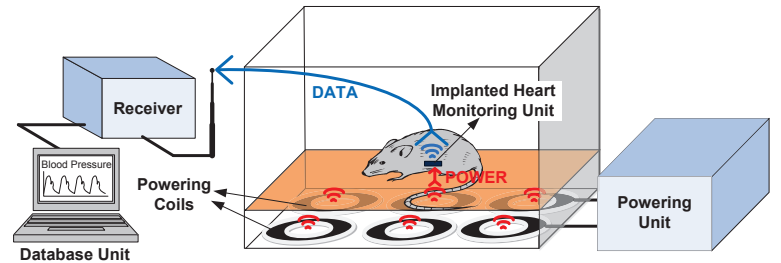

Fig. 2. Scenario for remotely powered implantable heart monitoring system for freely moving animal

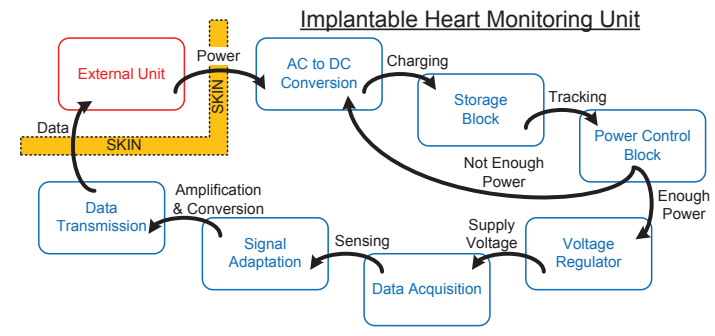

Fig. 3. Functional block diagram of implantable heart monitoring system

is an efficient method for short-range power transmission. The power is transferred to the implanted system by an array of powering coils under the animal cage. These coils are powered by a suitable control mechanism which turns on the appropriate coils and off the redundant coils by tracking the animal position in the cage [7]. Accordingly, the overall power consumption of the external unit is decreased and also the animal is disturbed minimally by turning on only the appropriate coil which keeps the animal cage at reasonable temperature.

\section{BUILDING BLOCKS}

The overall system consists of external and implantable units. The external unit is composed of power amplifier to drive the powering coil and the receiver to receive the data from the implanted unit. The implanted unit consists of the power management blocks and the data acquisition and transmission blocks. Fig. 3 shows the functional block diagram of implantable heart monitoring system. In order to reduce the overall power consumption of the implanted unit, the design is kept as simple as possible.

The power is transmitted from the powering coil to the implanted coil. The coupling between the coils change due to the lateral misalignment. The received power is maximized when the implanted coil is placed on the center of the powering coil. In addition, the received power decreases and goes to zero when the implanted coil moves from the center to the edges of the powering coil. Therefore, the received power level is not constant and it changes due to the position of animal. In addition, the overall power consumption of the implanted unit is quite high and it needs a large capacitance value to operate when the power transmission is insufficient. Therefore, this study propose a charging technique which makes the implanted unit autonomous for changing received power levels. This technique allows to activate the implanted unit to measure the

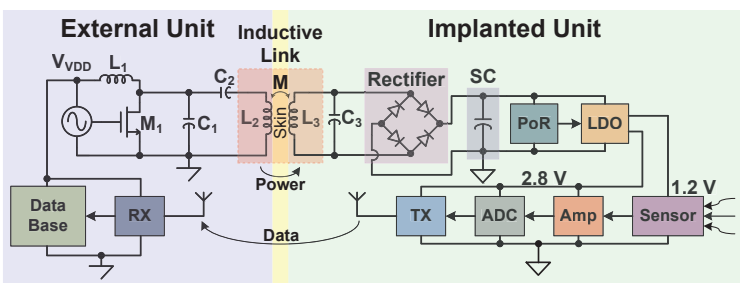

Fig. 4. Block diagram of implantable heart monitoring system

pressure and transmit the data. The received energy charges a storage capacitor. The voltage level on the capacitor is tracked by a power control block. The power control block enables the voltage regulator when the power level is enough to turn on the sensor and the transmitter blocks [8]. If the power level is not enough, the power control block disables the voltage regulator to create supply voltage for the sensor and the transmitter and allows to charge the storage capacitor by using received energy.

\section{A. Wireless Power Transmission}

Fig. 4 illustrates the block diagram of the implantable heart monitoring system. The power is amplified by an efficient class-E power amplifier at $8 \mathrm{MHz}$. The power is transmitted wirelessly over an optimized inductive link. The induced signal is rectified by a passive full-wave rectifier by connecting four diodes in H-bridge configuration. The implantable unit needs a large capacitance value to continue its operation. Therefore the rectified energy is stored in a $0.33 \mathrm{~F}$ supercapacitor (SC) [9]. The supercapacitor has quite large number of charge/discharge cycle compared to a rechargeable battery. In addition, the rechargeable battery needs a stable charging voltage which increase the complexity of design.

The voltage level on the supercapacitor is monitored by a Power on Reset (PoR) circuit [10]. The PoR manages the current drawn from the supercapacitor according to the charge on the capacitor. The PoR prevents the current when the supply voltage is insufficient. Accordingly, the PoR enables and disables the regulator when the supercapacitor is charged to $4.4 \mathrm{~V}$ and discharged to $3.3 \mathrm{~V}$, respectively. Therefore, the PoR creates a hysteresis loop between turning on and off the voltage regulator which defines an operation time for $2.5 \mathrm{~mA}$ load current as:

$$
\Delta t=\frac{\left(V_{H}-V_{L}\right) C_{S C}}{I_{L}}=\frac{(4.4-3.3) 0.33}{2.510^{-3}}=145 \mathrm{~s}
$$

where $\Delta t, C_{S C}, I_{L}$ is the operation time, the capacitance value of the supercapacitor, and the load current, respectively. $V_{H}$, and $V_{L}$ represent the charging and discharging voltage levels. The duration assures to complete the sensing of the blood pressure and transmitting the data. In order to generate clean and stable voltage supply for the sensor and the other blocks, a low-drop out voltage regulator (LDO) is used. The LDO has two output voltages $1.2 \mathrm{~V}$ and $2.8 \mathrm{~V}$ [11]. The $1.2 \mathrm{~V}$ is used for pressure sensor to reduce the overall power consumption of the system. 


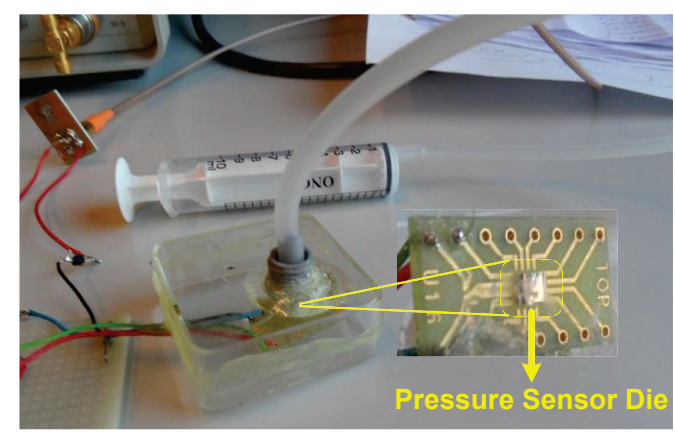

Fig. 5. Pressure sensor die and test setup

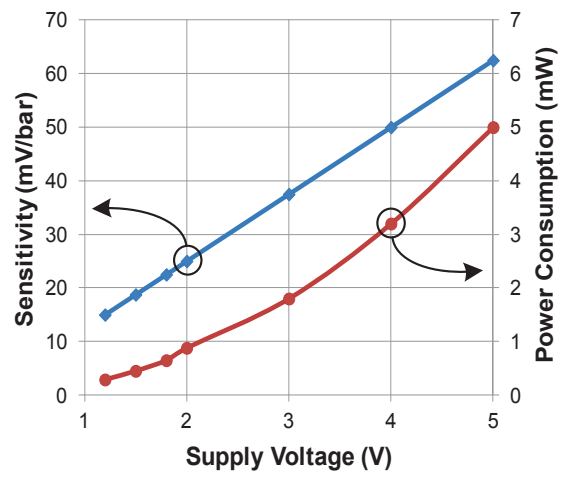

Fig. 6. Sensor sensitivity and power consumption versus supply voltage

\section{B. Pressure Sensor \& Data Acquisition}

The blood pressure in the left ventricle of the heart is measured by a silicon pressure sensor die [12] as shown in Fig. 5. The sensor is built as a piezoresistive bridge which converts the stress on the die to electrical signal to measure the pressure and has 2 bar full-scale range. This range is highly sufficient to measure LVP signal which changes from 1 bar to 1.3 bar. The sensor is characterized by using the test setup as shown in Fig. 5. Accordingly, Fig. 6 illustrates the sensitivity of the sensor proportional to the supply voltage. However, the power consumption of the sensor also increases with the supply. In order to reduce the power consumption, $1.2 \mathrm{~V}$ supply is applied to the sensor and $15 \mathrm{mV} / \mathrm{bar}$ of sensitivity is obtained. Nevertheless, this low sensitivity issue of the sensor is solved by a micropower instrumentation amplifier [13]. The amplifier has a voltage gain of 100 and also adapts the sensor output to $2.8 \mathrm{~V}$ supply voltage. The amplified signal is delivered to an Analog to Digital Converter (ADC) to convert the analog signal to the digital bits [14]. The ADC has 12 bit resolution which gives $0.68 \mathrm{mV}$ sensitivity at $2.8 \mathrm{~V}$ supply voltage. The output of the amplifier changes $450 \mathrm{mV}$ for 0.3 bar pressure difference. Therefore the pressure resolution is 0.45 mbar. The output data rate of the ADC is $125 \mathrm{~Hz}$ and each data contains 24 bits which include 12-bit conversion result, header and status bits. The ADC needs an external clock reference. Therefore, the reference clock is obtained by a micro-power oscillator [15].

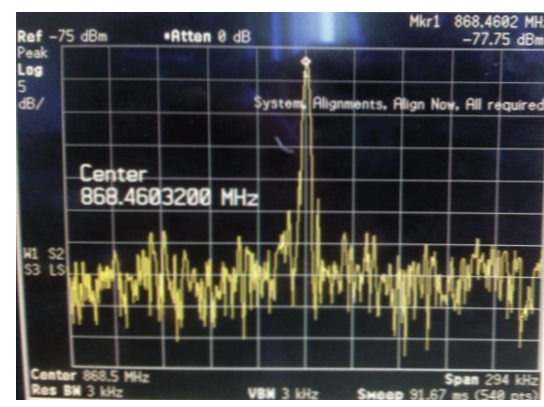

Fig. 7. Frequency spectrum of transmitter

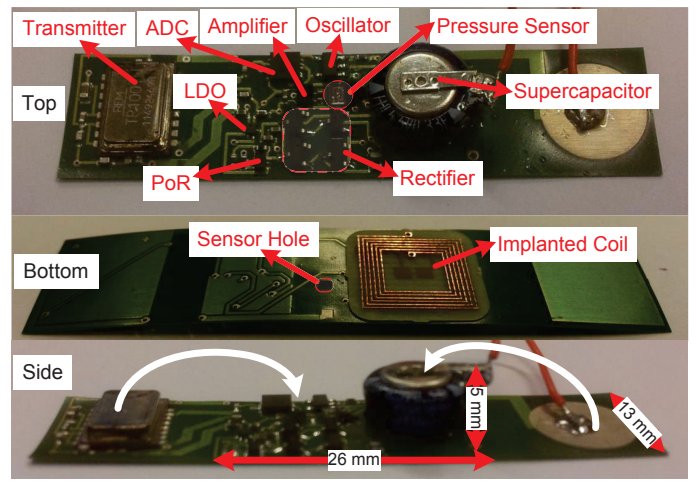

Fig. 8. Remotely powered implantable heart monitoring unit

\section{Data Transmission}

The output of the ADC is connected to transmitter. The digital bits are transmitted by a OOK modulated transmitter at $868 \mathrm{MHz}$ [16]. Fig. 7 shows the frequency spectrum of the transmitter during sending bit "1". The signal power is around $-78 \mathrm{dBm}$ at $40 \mathrm{~cm}$ away from the transmitter. In order to achieve the data communication, the receiver is designed by using same transceiver [16]. The receiver has $-95 \mathrm{dBm}$ sensitivity with a whip antenna. Finally, the data is delivered to the database unit to reconstruct the blood pressure signal.

\section{Measurement Results}

In order to prove the concept, the implantable unit blocks are assembled on a flexible Kapton substrate which is 100 $\mu \mathrm{m}$ of thickness. Fig. 8 demonstrates the remotely powered implantable heart monitoring unit. The discrete components are placed on the top of the Kapton substrate as shown in Fig. 8. Additionally, all the circuits are chosen by considering to achieve not only low power consumption but also small volume. Moreover, the left and right sides of the implanted unit where the transmitter and ground plane are placed are folded over other circuits as shown in Fig. 8 (Side). Accordingly, the overall size of the implantable unit is squeezed to $26 \times 13 \mathrm{~mm}$ with $5.5 \mathrm{~mm}$ height. The $12 \times 12 \mathrm{~mm}$ implanted coil is also attached to the bottom of the implantable unit.

The power is transmitted over $25 \mathrm{~mm}$ distance at $8 \mathrm{MHz}$ by using the class-E amplifier and the powering coil. The induced signal is rectified by Schottky diodes which have 0.4 $\mathrm{V}$ forward voltage at $10 \mathrm{~mA}$ load current. The supercapacitor 


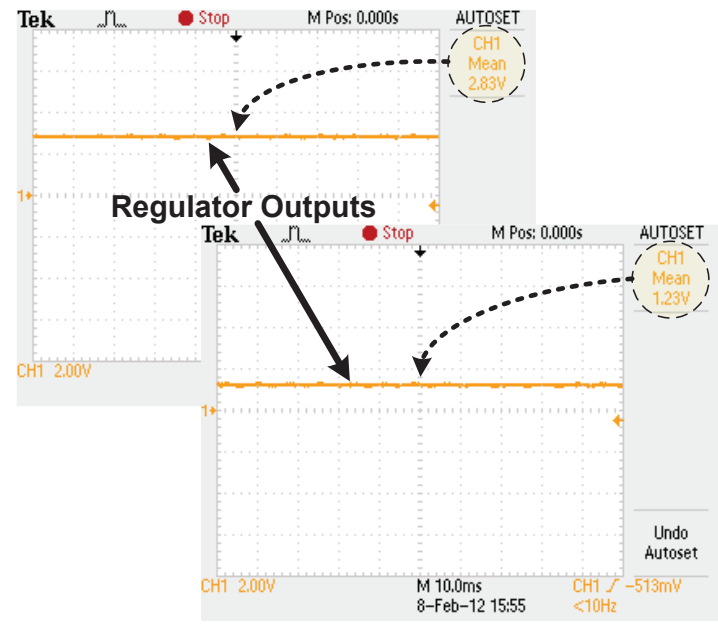

Fig. 9. Outputs of voltage regulator

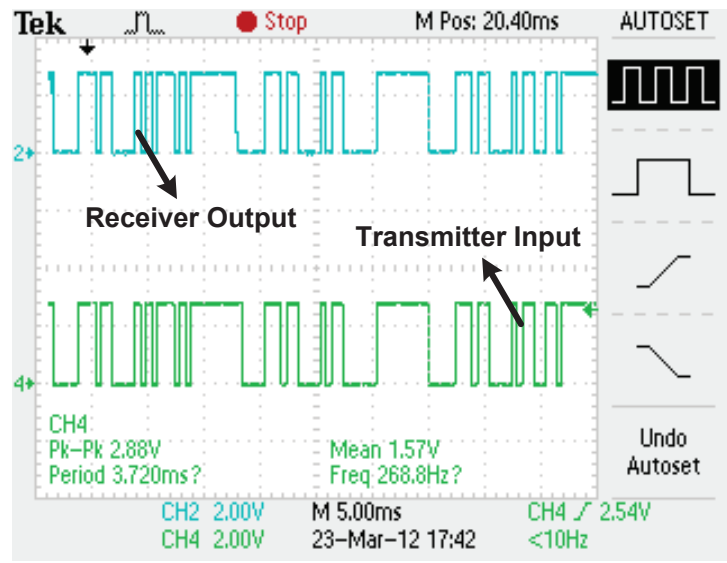

Fig. 10. Data communication

is charged by this diode bridge. When the voltage level on the supercapacitor is reached to $4.4 \mathrm{~V}$, the PoR enables the LDO and the LDO supplies two regulated output voltages 1.2 $\mathrm{V}$ and $2.8 \mathrm{~V}$ as shown in Fig. 9. The PoR is turned off by the PoR when the voltage level on the supercapacitor reduces to $3.3 \mathrm{~V}$. The power consumption of the sensor is $1.8 \mathrm{~mW}$ at 3 $\mathrm{V}$ supply. Therefore, the sensor is supplied by $1.2 \mathrm{~V}$ supply to reduce the power consumption. The power consumption of the sensor is $0.288 \mathrm{~mW}$ at $1.2 \mathrm{~V}$ supply voltage.

The oscillator generates $10 \mathrm{kHz}$ clock signal which is used as external reference clock of the ADC. Although the reference clock is fast enough, the ADC completes the data conversion in $8 \mathrm{~ms}$. Therefore, the data rate is limited with $125 \mathrm{data} / \mathrm{s}$. The transmitter offers OOK modulation which reduces the average power consumption by turning off the transmitter when the bit " 0 " is sent. Therefore, the serial data output of the ADC is connected directly to the transmitter. The data rate of the transmitter increases up to $30 \mathrm{kbps}$. The transmitter has 1.75 $\mathrm{mA}$ current consumption at $2.8 \mathrm{~V}$ supply when it is turned on. The overall power consumption of the implanted unit is around $7 \mathrm{~mW}$.

\section{Conclusion}

A remotely powered implantable heart monitoring system for freely moving animals is presented. This implantable sensor measures the blood pressure in the left ventricle and transmits the data to a database unit to monitor the animal. LVP signal is monitored by the piezoresistive sensor die. The power is transmitted wirelessly at $8 \mathrm{MHz}$ over $25 \mathrm{~mm}$ distance by using the magnetically coupled coils. The power management block which consists of the full-wave rectifier, the supercapacitor, the PoR, and the LDO introduces an autonomy to the implanted unit for changing received power level due to the freely moving animals. The pressure signal is converted to digital bits by using the ADC. The data is transmitted by the OOK modulated transmitter at $868 \mathrm{MHz}$. The overall power consumption of the implanted unit is $7 \mathrm{~mW}$. The implanted unit is obtained by assembling all the discrete components on a thin flexible Kapton substrate. The overall size of the implanted unit is reduced to $26 \times 13 \times 5.5 \mathrm{~mm}$.

\section{ACKNOWLEDGMENT}

The authors thank to O. Atasoy, G. Yilmaz, T. Guo, and K.M. Silay for their valuable supports. This project is supported by Swiss National Funding (SNF) through Sinergia Initiative and SSSTC-EG56-092011.

\section{REFERENCES}

[1] H.J. Adrogue, N.E. Madias. "Sodium and potassium in the pathogenesis of hypertension." in N. Engl. J. Med., 2007, vol. 356, pp. 1966-1978.

[2] WHO. (2008). World Health Statistics 2008 [Online]. Available: http://www.who.int/gho/publications/world_health_statistics/2008.

[3] D.J. Young, "An RF-powered wireless multi-channel implantable biosensing microsystem," in Proc. IEEE Conf. Eng. Med. Biol. Soc., 2010, pp. 6413-6416.

[4] E.G. Kilinc, et. al., "Remotely powered telemetry system with dynamic power-adaptation for freely moving animals," in IEEE BioCAS 2012 2012, pp. 260-263.

[5] Q. Wang, H.R. Brunner, M. Burnier, "Determination of cardiac contractility in awake unsedated mice with a fluid-filled catheter," in Am. J. Physiol. Heart Circ. Physiol., 2004, vol.286, no.2, pp. H806-814.

[6] Q. Wang, et. al., "Impact of salt on cardiac differential gene expression and coronary lesion in normotensive mineralocorticoid-treated mice," in Am. J. Physiol. Regul. Integr. Comp. Physiol., 2012, vol. 302, no. 9, pp. R1025-1033.

[7] E.G. Kilinc, et. al., "Intelligent cage for remotely powered freely moving animal telemetry systems," in Proc. IEEE ISCAS 2012, 2012, pp. 22072210 .

[8] E.G. Kilinc, C. Dehollain, "Intelligent Remote Powering," EPO Patent 12180919.8, August 17, 2012.

[9] Cornell Dubilier Electronics, Inc., USA. EDLSD334V5R5C - 0.33 F, 5.5 V, EDL Supercapacitor [Online]. Available: http://www.cde.com.

[10] Maxim Integrated Products, Inc., USA. MAX6777-LT - Low Power, 1\%-Accurate Battery Monitors [Online]. Available: http://www. maximintegrated.com.

[11] Micrel, Inc., USA. MIC5380-M4YFT - High Performance Dual 150mA LDO [Online]. Available: http://www.micrel.com.

[12] Silicon Microstructures, Inc., USA. SM5112-030A - Harsh Environment Absolute Silicon Pressure Die [Online]. Available: http://www.simicro.com.

[13] Analog Devices, Inc., USA. AD8235 - $40 \mu$ A Micropower Instrumentation Amplifier [Online]. Available: http://www.analog.com.

[14] Analog Devices, Inc., USA. AD7170 - 12-Bit Low Power Sigma-Delta ADC [Online]. Available: http://www.analog.com.

[15] Linear Technology, Corp., USA. LTC6906 - Micropower, 10kHz to $1 \mathrm{MHz}$ Resistor Set Oscillator [Online]. Available: http://www.linear.com.

[16] RFM, Inc., USA. TR1001 - 868.35 MHz Hybrid Transceiver [Online]. Available: http://www.rfm.com. 